\title{
Management of Retinal Detachment Accompanied by PVR: Controversy Continues
}

\section{Guerra Garcia RA*}

A member of the Retina-Vitreous Service at the InstitutoCubano de Oftalmología and a research associate at the University of La Habana, Cuba

*Corresponding author: Roberto Alejandro Guerra Garcia, A member of the Retina-

\section{Case Report}

Volume 2 Issue 5

Received Date: November 29, 2017

Published Date: December 27, 2017

Vitreous Service at the Instituto Cubano de Oftalmología and a research associate at the University of La Habana, Cuba, Email: ralejandrogg2011@gmail.com

\section{Abstract}

Even with the new advances in vitreous-retinal surgery achieved in recent years, vitreous surgeons still have to weigh the likelihood of needing more than one surgery to treat rhegmatogenous retinal detachment (RRD). This situation becomes more likely if the detachment is accompanied or there is a risk of proliferative vitreoretinopathy (PVR). PVR has been described as a process analogous to the healing and remodeling of the retina and its adjacent structures. This condition is found in approximately $10 \%$ of RRD cases.

The migration of pigment epithelial cells through the breaks present in the RRD is well known [1-3]. Glial proliferation can be observed from micro-ruptures in the internal limiting membrane secondary to the vitreous traction or even to the peeling of membranes in previous interventions, in the case of recurrent RRD. These phenomena lead to migration and proliferation of cells above and below the retina with the formation of membranes [4-6]. Finally, the contraction of these cells is added due to the presence of intracellular microtubules similar to those existing in muscle cells.

The traction on the retina exerted by these membranes plays an important role in the loss of the normal transretinal pressure gradient. Aggravating this phenomenon already present due to passage of fluid to the sub-retinal space through the retinal breaks. Therefore, when the surgeon is faced with RRD + PVR cases, he must take into account not only the main objective of stopping the fluid flow through the breaks, but also face and eliminate all traction on the retina.

With this article, we attempt to offer elements found in the consulted bibliography, accompanied by our personal assessment of this condition, based on our experiences obtained from daily practice with our patients.

Keywords: Retinal Detachment; Rhegmatogenous; Vitreoretinopathy; Pseudophakic; Phacoemulsification 


\section{Open Access Journal of Ophthalmology}

Abbreviations: RRD: Rhegmatogenous Retinal Detachment; PVR: Proliferative Vitreoretinopathy; PPV: Pars Plana Vitrectomy; IOL: Intraocular Lens; PFO: Perfluoro-Octane.

\section{Counseling the Patient and their Relatives}

The individual and the family can often have the misperception that having a retinal condition has a very poor prognosis. On the other hand, on occasions, they may have very high expectations due to knowledge from more frequent and simple ocular affections that lead to systematized surgeries with high visual and anatomical success rates. The surgeon must personalize each case and explain the characteristics, treatment and prognosis of the condition in an accessible language.

\section{Lens Status}

The patient planned for pars plana vitrectomy (VPP) due to RRD + PVR should ideally be pseudophakic or aphakic. Complicated maneuvers and prolonged surgical times can lead to iatrogenic lens opacification during surgery, which creates visualization problems and jeopardizes the outcome. As some authors suggest, the extraction of the lens allows a meticulous approach to the peripheral vitreous and an anterior dissection of the PVR.

In the case of planning the removal of the lens, the technique of choice is phacoemulsification, which can be performed prior or during PPV [7]. The calculation of the intraocular lens (IOL) in the presence of complex detachments can be difficult, even with modern means. If there is any doubt, it is advisable to leave the patient aphakic. Some authors recommend the elimination of the posterior capsule to avoid it being used as "scaffolding" for reproliferation, endangering the eye with hypotonia due to damage to the ciliary body.

\section{Bands and Scleral Implants}

Surgery with scleral implants can be used alone in cases of RRD that meet the criteria for it indication, being accompanied by localized and inactive PVR [8]. However, with the development of PPV, scleral implants, especially silicone bands, are used as an adjunct to PPV. The surrounding band should be placed leaving little height. Non-uniform depression (not in 360o) caused by a sponge or implant will induce astigmatism. It is important to bear in mind that these patients could be already operated from cataract with IOL implantation taking into account the previous refractive state.

The anterior PVR tends to be more frequent in the lower quadrants due to the force of gravity, the upright posture of the individual when walking, sitting and the buoyancy of the tamponades used in these surgeries. The union of these factors favors the persistence of a lower aqueous meniscus that compartmentalizes the cells and inflammatory mediators in the lower quadrants). Therefore, it is frequent the need to perform extensive inferior circular retinectomies, generally leaving the periphery of the superior sectors more conserved, having as an advantage that tamponade acts more effectively in these higher areas $[9,10]$. This is the reason why we do not generally use bands when performing large retinectomies (close to 180o) [11,12]. In fact, on several occasions when faced RRD with PVR previously operated with PPV + external band, we decided to withdraw the band (assessing risk / benefit) because of it negative action due to the resulting cleavage effect when the retinectomy is in the posterior slope (especially if the band was left very tight). This, accompanied by a certain rigidity of the retina, facilitates the passage of fluid into sub retinal space.

In general, given the unpredictable complexity of the maneuvers that will be required to manage the PVR when evaluating the patient in the office and other aspects discussed above, the tendency of the authors of this work has been to gradually reduce the use of scleral surgery in all its variants for these cases. We strongly believe that the high rates of anatomical success reported by other authors when using scleral surgery as a primary technique, or PPV as a technique in the second intervention in cases previously operated only with scleral implants, is due to the uncomplicated nature of these RRD, in comparison with those that go directly to PPV. Having less importance the aid that should be expected from a circular band [13].

It should not be forgotten that there are previous studies whose results do not show a significant improvement in anatomical success when scleral implants and PPV are associated in the presence of inferior retinectomies. Showing no difference when using VPP alone.

\section{Vitrectomy and Membrane Peeling}

It is essential to perform vitrectomy as complete as possible, starting with the condensations that are usually 


\section{Open Access Journal of Ophthalmology}

present in the vitreous base and behind the lens [7]. VPP is recommended in an "anterior-posterior" fashion, including posterior vitreous detachment. During this period, surgeon should take aid from staining techniques (triamcinolone, trypan blue, Etc.) to visualize vitreous filaments and modern vitrectomy machines, trying to reduce traction on the retina by managing the aspiration flow at high cut rates.

Once the vitreous is removed, we proceed to the peeling of the pre and sub-retinal membranes that could be present. This is the time to take your time and peel off as much tissue as possible, keeping in mind that all this effort can spare our patient from retinectomies. Always remembering the sphericity of the eye, pre-retinal membranes should be approached with "in-out" manner, away from the optic nerve. Using vitreo-retinal forceps, trying to find a zone of cleavage and without damaging the underlying retina. The sub-retinal membranes can be approached directly or performing a peripheral retinotomy, approaches the membrane sub-retinally.

With the technology available, it is becoming less necessary to use scissors and pikes for these cases.

\section{The Reattachment Experiment \& Retinectomy's Decision Process}

Many aspects discussed in this section have points of agreement with the philosophy of Steve Charles M.D., who is owed many of the maneuvers and terms that we use in modern vitreous surgery.

Once the vitrectomy is completed with the delamination / segmentation / peeling of the membranes, fluid-air exchange is performed. Then we proceed to test if the retina flattens with the actions done so far. With the advent of heavy liquids, this maneuver can be assisted with perfluoro octanes liquids (PFO). The author recommends making a sandwich with PFO to protect the posterior pole but not to surpass the peripheral area where the tears are found, to avoid heavy liquid getting under the retina if residual traction remains. The peripheral part of the vitreous cavity is filled with air and then the reattachment experiment is carry out (Figure 1). Performing this maneuver under air take advantage of its greater surface tension with respect to the PFO (73 dynes / cm vs. 50 dynes / cm approximately). The presence of sub retinal air is an unequivocal sign of residual traction.

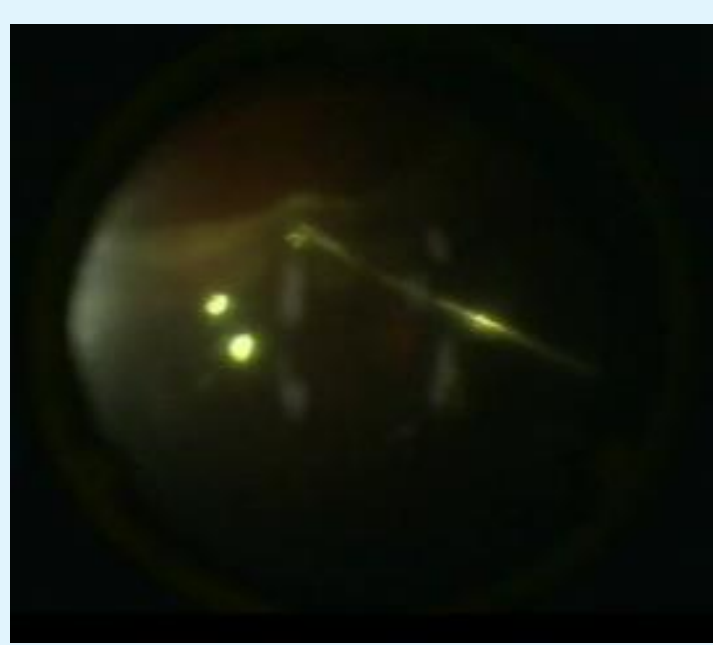

Figure 1: Making the reattachment experiment. Touching the retina with the cutter under air: is evident that some stiffness and traction is remaining.

\section{The Retinectomy in Detail}

Retinectomy should be oriented according to the force vectors we want to disrupt. The peripheral and circular are very frequent, being able to surpass $180 \mathrm{o}$, but sometimes doing it radially (especially when there is a circular contraction of the vitreous base) the results are surprising.

It is always important to remove the peripheral retina from the incision made [14]. This detail makes the difference from the retinotomy recommended by some surgeons, since it is common to observe chronic hypotonia, detachments of the ciliary body and peripheral neovascularizations associated with this last procedure.

Many authors recommend the use of diathermy to prevent bleeding and delineate the edge of the retina [15]. Sometimes it is advantageous to treat only the most important vessels with diathermy, leaving untreated spaces, but drawing a line of perceptible dots as guide [16]. It is common to see contracture of the edge of the retinectomy associated with excessive cauterization of the retina with traces of stiffness due to unpeeled membranes, after being excised with the vitrectome. (See video https://eyetube.net/video/uungdav//) On the other hand, in peripheral areas it is possible to perform this procedure directly with the cutter without using 


\section{Open Access Journal of Ophthalmology}

cauterization. This is why we prefer to perform this step under air, taking advantage of it greater surface tension, ensuring better hemostasis. It is important to treat any bleeding, since theresidual clot has high potential to form recurrent PVR. (See video http://eyetube.net/video/uudfroc/) The retinectomy will be extended (staying peripheral and towards the ora) until the retina is completely flattened. Emphasis should be placed on cutting the remnant of the vitreous at the edges of the retinectomy near the ora. To achieve this goal, it is practical to use the so-called interface vitrectomy. Once again, under the air the cutter can be used thanks to the aqueous film that is confined to the vitreous base while it is buffered by air, PFO or even silicone oil (Figure 2).

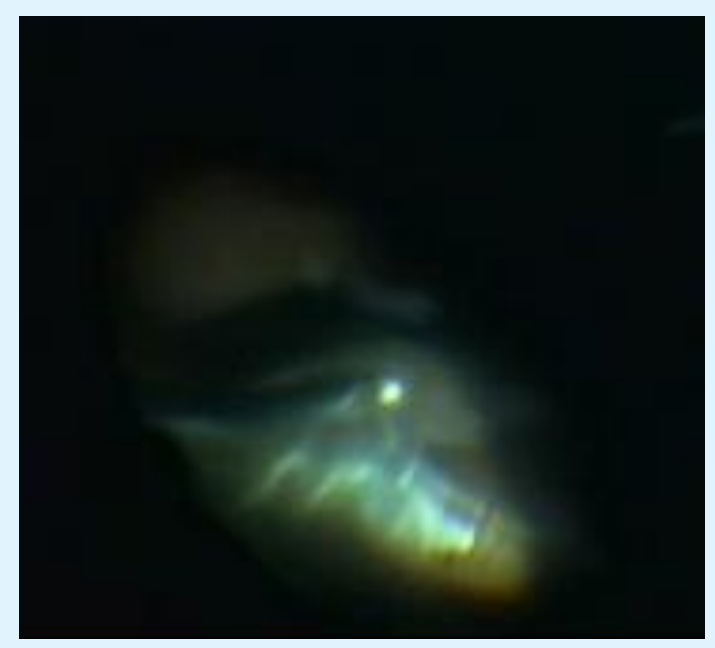

Figure 2: Interface vitrectomy: the retinectomy's edges are remodeled and shaved using the cutter through the aqueous interface confined between the air and PFO.

\section{Using the Laser Properly}

Some experienced surgeons recommend not performing laser treatment on the created retinectomy, or treat very lightly to avoid the process of re-proliferation and contracture associated with the inflammatory reaction that the laser could cause.

In our practice, we perform two or three rows of laser with moderate and quite confluent effect, since it is probable that this undesired effect of the contracture is due to an intense diathermy previously applied and not to laser treatment. This process can be carried out preferably under air or after an "overfill" of PFO.

Very weak laser may not help restore adhesion to the pigmented epithelium in a probably thickened and rigid retina. Very intense laser could cause hemorrhage and iatrogenic micro-holes.

\section{Choosing the Agent for Tamponade}

As we talk about cases in which the first surgery has failed and where extensive and possibly inferior ruptures / retinectomies are frequent, the long-acting tampons should be taken into account.

The presence in the market of increasingly pure and dense silicone oils has come to solve this problem by delaying the emulsion process. That is why we prefer this option in these cases, often making a direct exchange of PFO-silicone oil, if the situation required overfilling of PFO. In our hands, using valvedtrocar systems, this form of filling is faster than if PFO-air-silicone oil exchange is performed. In addition, it makes it less possible for any aqueous residue to pass under the retina through the large existing tears.

\section{Conclusion}

Much has been written and discussed on the controversial issue of RRD surgery accompanied by PVR, however, we suggest to vitreous surgeons who face these cases to use a systematized strategy that incorporates the knowledge acquired from different sources. Using in each step of the surgery the approach that works best in your hands depending on the situation you face.

\section{References}

1. Charteris DG, Sethi CS, Lewis GP, Fisher SK (2002) Proliferative vitreoretinopathy-developments in adjunctive treatment and retinal pathology. Eye (Lond) 16(4): 369-374.

2. Tseng W, Cortez RT, Ramirez G, Stinnett S, Jaffe GJ (2004) Prevalence and risk factors for proliferative vitreoretinopathy in eyes with rhegmatogenous retinal detachment but no previous vitreoretinal surgery. Am J Ophthalmol 137(6): 1105-1115.

3. Heimann H, Bartz-Schmidt KU, Bornfeld N, Weiss C, Hilgers RD (2007) Scleral buckling versus primary vitrectomy in rhegmatogenous retinal detachment: a prospective randomized multicenter clinical study. Ophthalmology 114(12): 2142-2154.

4. Campochiaro PA, Glaser BM (1985) Platelet-derived growth factor is chemotactic for human retinal 


\section{Open Access Journal of Ophthalmology}

pigment epithelial cells. Arch Ophthalmol 103(4): 576-579.

5. Campochiaro PA (1997) Pathogenic mechanisms in proliferative vitreoretinopathy. ArchOphthalmol 115(2): 237-241.

6. Pastor JC (1998) Vitreorretinopatía proliferativa: una visión general. Surv Ophthalmol 43: 3-18.

7. Quiram PA, Gonzales CR, Hu W, Gupta A, Yoshizumi MO, et al. (2006) Outcomes of vitrectomy with inferior retinectomy in patients with recurrent rhegmatogenous retinal detachments and proliferative vitreoretinopathy. Ophthalmology 113 (11): 2041-2047.

8. Kuhn F (2008) Ocular Traumatology. New York: Springer.

9. Sigler EJ, Randolph JC, Calzada JI, vitrectomía de Charles S (2013) Pars plana con perfluoro-N-octano postoperatorio a mediano plazo para desprendimiento de retina inferior recurrente complicado por vitreorretinopatía proliferativa avanzada. Retina 33 (4): 791-797.

10. Charles S, Randolph JC, Sigler EJ (2012) Reintervención de la vitrectomía de Pars plana sin eliminar el aceite de silicona. Retina 32 (8): 16641665.
11. Lu L, Gao R, Lin X (1998) The application of retinotomy and retinectomy for complicated retinal detachment. Yan Ke Xue Bao 14(2): 94-96.

12. Shalaby KA (2010) Relaxing retinotomies and retinectomies in the management of retinal detachment with severe proliferative vitreoretinopathy (PVR). Clin Ophthalmol 4: 11071114.

13. Mancino R, Aiello F, Ciuffoletti E, Di Carlo E, Cerulli A, et al. (2015) Inferior retinotomy and silicone oil tamponade for recurrent inferior retinal detachment and grade C PVR in eyes previously treated with pars plana vitrectomy or scleral buckle. BMC Ophthalmol 15: 173.

14. Zhaoxin J, Qiu S, Lou B, Lin M, Tan J (2016) Radial Retinotomies with Endodiathermy for Severe Proliferative Vitreoretinopathy: Short-Term Results. Journal of Ophthalmology 2594574: 9 pages.

15. Bourke RD, Cooling RJ (1996) Vascular consequences of retinectomy. Arch Ophthalmol 114(2): 155-160.

16. John D. Pitcher (2017) Tips and tricks for polishing your retinectomy skills. Retina Today 12(4): 35-37. 